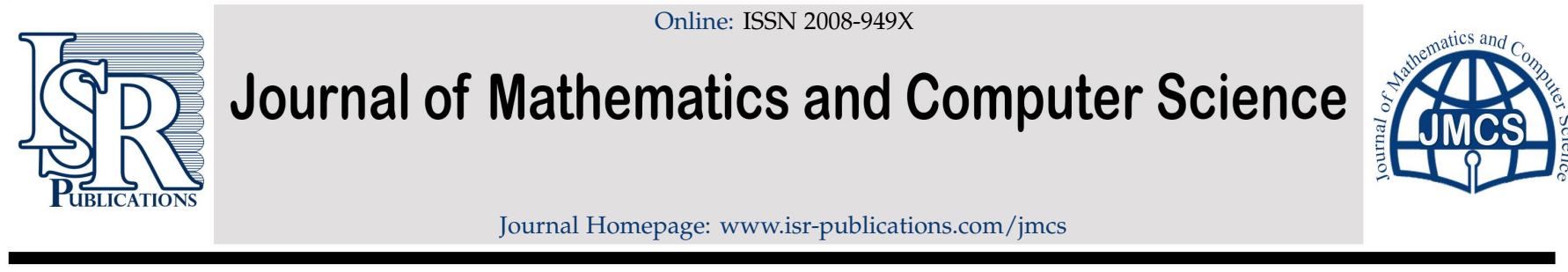

\title{
Explicit Halpern-type iterative algorithm for solving equi- librium problems with applications
}

\author{
Kanikar Muangchoo \\ Faculty of Science and Technology, Rajamangala University of Technology Phra Nakhon (RMUTP), 1381 Pracharat 1 Road, \\ Wongsawang, Bang Sue, Bangkok 10800, Thailand.
}

\begin{abstract}
A number of iterative algorithms have been established to solve equilibrium problems, and one of the most effective methods is a two-step extragradient method. The main objective of this study is to introduce a modified algorithm that is constructed around two methods; Halpern-type method and extragradient method with a new size rule to solve the equilibrium problems accompanied with pseudo-monotone and Lipschitz-type continuous bi-function in a real Hilbert space. Using certain mild conditions on the bi-function, as well as certain conditions on the iterative control parameters, proves a strong convergence theorem. The proposed algorithm uses a monotonic step size rule depending on local bi-function information. The main results are also used to solve variational inequalities and fixed-point problems. The numerical behavior of the proposed algorithm on different test problems is provided compared to other existing algorithms.
\end{abstract}

Keywords: Equilibrium problem, Lipschitz-type continuity, strong convergence, fixed point problem, variational inequality problem.

2020 MSC: 47J25, 47H09, 47H06, 47J05.

(C)2022 All rights reserved.

\section{Introduction}

Suppose that $\mathcal{C}$ is a non-empty convex and closed subset of a real Hilbert space $\mathcal{H}$. Let $f: \mathcal{H} \times \mathcal{H} \rightarrow \mathbb{R}$ be a bi-function satisfying $f\left(y_{1}, y_{1}\right)=0$, for each $y_{1} \in \mathcal{C}$. An equilibrium problem $[7,11]$ for $f$ on the set $\mathcal{C}$ is defined in the following way:

$$
\text { Find } \mathrm{u}^{*} \in \mathcal{C} \text { such that } f\left(\mathrm{u}^{*}, \mathrm{y}_{1}\right) \geqslant 0, \forall \mathrm{y}_{1} \in \mathcal{C} \text {. }
$$

Furthermore, a solution set of equilibrium problem over the set $\mathcal{C}$ is denoted by $\beth_{\text {Ep }}$ and $u^{*}$ is an any arbitrary element of $\beth_{\mathrm{Ep}}$.

The problem (EP) is a general mathematical problem in the sense that it carries together many mathematical problems, i.e., the fixed point problems, the vector and scalar minimization problems, the problems of variational inequalities, complementarity problems, problems of the saddle point, the Nash equilibrium problems in non-cooperative games and the inverse optimization problems $[7,28]$. The problem

Email address: kanikar.m@rmutp.ac.th (Kanikar Muangchoo)

doi: $10.22436 /$ jmcs.025.02.02

Received: 2020-11-08 Revised: 2021-01-29 Accepted: 2021-04-15 
(EP) is also known as the well-known Ky Fan inequality due to the previous contribution [11]. Due to the importance of this problem (EP) and its applications in both pure and applied sciences, many researchers have been studied this topic in recent years [2, 6, 13, 30-36].

An important iterative method is introduced by Tran et al. [39] and iterative sequence $\left\{u_{n}\right\}$ has the following form:

$$
\left\{\begin{array}{l}
u_{0} \in \mathcal{C}, \\
y_{n}=\underset{y \in \mathcal{C}}{\arg \min }\left\{\zeta f\left(u_{n}, y\right)+\frac{1}{2}\left\|u_{n}-y\right\|^{2}\right\}, \\
u_{n+1}=\underset{y \in \mathcal{C}}{\arg \min }\left\{\zeta f\left(y_{n}, y\right)+\frac{1}{2}\left\|u_{n}-y\right\|^{2}\right\},
\end{array}\right.
$$

where $0<\zeta<\min \left\{\frac{1}{2 c_{1}}, \frac{1}{2 c_{2}}\right\}$. This method is also considered as the two-step extragradient method in [39] due to the previous contribution of the Korpelevich [23] extragradient method to solve saddle point problems. It is important to mention that previous established methods are operating by the use of constant step size depends upon Lipschitz-type constants as well as provides a weak convergence sequence $[12,15,25,39]$ and others in $[1,16-19,21,29,40]$.

In order to operate the method (1.1), previous knowledge of Lipschitz-type constants is required. Such Lipschitz-type constants are normally not known or difficult to compute. To overcome this drawback, Hieu et al. [20] established a method to solve equilibrium. The method has the following form:

$$
\left\{\begin{array}{l}
u_{0} \in \mathcal{C}, \\
y_{n}=\underset{y \in \mathcal{C}}{\arg \min }\left\{\zeta_{n} f\left(u_{n}, y\right)+\frac{1}{2}\left\|u_{n}-y\right\|^{2}\right\} \\
u_{n+1}=\underset{y \in \mathcal{C}}{\arg \min }\left\{\zeta_{n} f\left(y_{n}, y\right)+\frac{1}{2}\left\|u_{n}-y\right\|^{2}\right\}
\end{array}\right.
$$

where the step size rule $\left\{\zeta_{n}\right\}$ is revised as follows:

$$
\zeta_{n+1}= \begin{cases}\min \left\{\zeta_{n}, \frac{\mu\left(\left\|u_{n}-y_{n}\right\|^{2}+\left\|u_{n+1}-y_{n}\right\|^{2}\right)}{2\left[f\left(u_{n}, u_{n+1}\right)-f\left(u_{n}, y_{n}\right)-f\left(y_{n}, u_{n+1}\right)\right]}\right\}, & \text { if } f\left(u_{n}, u_{n+1}\right)-f\left(u_{n}, y_{n}\right)-f\left(y_{n}, u_{n+1}\right)>0 \\ \zeta_{0}, & \text { else. }\end{cases}
$$

However, the methods (1.1) and (1.2) generate weakly convergent iterative sequences in a real Hilbert space. A natural question that arises in the case of infinite-dimensional Hilbert spaces is how to create a method that gives strong convergence. The viscosity method [26] and hybrid (outer approximation) method [10] have been designed to answer this question. Very recently, Kraikaew and Saejung [24] have used the subgradient extragradient method in [9] and Halpern iteration in [14], and proposed the Halpern subgradient extragradient method to figure out variational inequality problems in Hilbert spaces. In the case of infinite-dimensional Hilbert spaces, it is always important to study strong convergence sequences.

The natural question that arises is: "Is it possible to modify the method (1.2) in the sense to obtain a strongly convergent sequence with a monotone step size rule"?

In this paper, we provide a positive answer to this question, i.e., the extragradient method provides a strong convergence sequence by using a monotonic step size rule for solving equilibrium problems accompanied by pseudo-monotone bifunction. Motivated by the works of Censor et al. [9] and Halpern method [14] we introduce new Halpern extragradient type method to solve the problem (EP) in the case of infinite-dimensional a real Hilbert spaces. The original result of Halpern [14] was used to solve the problem of finding a fixed point of a single operator and Bauschke [3] extended to that of finding a common fixed point of finitely many operators.

In particular, the key contributions in this paper are listed below:

- In this paper, we introduce an explicit Halpern subgradient method with a monotone step size rule to solve the equilibrium problem in a real Hilbert space. 
- In mild conditions, we have established a strong convergence result that is corresponding to the proposed method.

- Applications of our main results are studied in order to solve particular classes of equilibrium problems in real Hilbert spaces.

- We have provided a detailed numerical description of the proposed method to verify the theoretical results and comparing the results in [15, Algorithm 3.2]. Our numerical results indicate that our approach is more effective than the existing one.

The rest of this paper has been arranged as follows: Section 2 has some preliminary and necessary results. Section 3 consists of the described method and provides a strong convergence result. Section 4 consists of applications of our main results. Section 5 consists of the numerical examination of the proposed method compared to existing ones.

\section{Preliminaries}

Suppose that $\mathcal{C}$ be a non-empty convex and closed subset of a real Hilbert space $\mathcal{H}$. We consider different types of a bi-function monotonicity (see $[5,7]$ for more details). A bi-function $f: \mathcal{H} \times \mathcal{H} \rightarrow \mathbb{R}$ on $\mathcal{C}$ for $\gamma>0$ is said to be

(f1) $\gamma$-strongly monotone if

$$
f\left(y_{1}, y_{2}\right)+f\left(y_{2}, y_{1}\right) \leqslant-\gamma\left\|y_{1}-y_{2}\right\|^{2}, \forall y_{1}, y_{2} \in \mathcal{C}
$$

(f2) monotone if

$$
f\left(y_{1}, y_{2}\right)+f\left(y_{2}, y_{1}\right) \leqslant 0, \forall y_{1}, y_{2} \in \mathcal{C}
$$

(f3) $\gamma$-strongly pseudo-monotone if

$$
f\left(y_{1}, y_{2}\right) \geqslant 0 \Longrightarrow f\left(y_{2}, y_{1}\right) \leqslant-\gamma\left\|y_{1}-y_{2}\right\|^{2}, \forall y_{1}, y_{2} \in \mathcal{C}
$$

(f4) pseudo-monotone if

$$
f\left(y_{1}, y_{2}\right) \geqslant 0 \Longrightarrow f\left(y_{2}, y_{1}\right) \leqslant 0, \forall y_{1}, y_{2} \in \mathcal{C} .
$$

From the above definition the following consequences are obvious:

$$
(\mathrm{f} 1) \Longrightarrow(\mathrm{f} 2) \Longrightarrow(\mathrm{f} 4) \text { and }(\mathrm{f} 1) \Longrightarrow(\mathrm{f} 3) \Longrightarrow(\mathrm{f} 4) \text {. }
$$

Generally, the converse implication does not hold. A bi-function $f: \mathcal{H} \times \mathcal{H} \rightarrow \mathbb{R}$ is said to be Lipschitztype continuous on $\mathcal{C}$ if there exists two constants $c_{1}, c_{2}>0$ such that

$$
f\left(y_{1}, y_{3}\right) \leqslant f\left(y_{1}, y_{2}\right)+f\left(y_{2}, y_{3}\right)+c_{1}\left\|y_{1}-y_{2}\right\|^{2}+c_{2}\left\|y_{2}-y_{3}\right\|^{2}, \forall y_{1}, y_{2}, y_{3} \in \mathcal{C} .
$$

The normal cone of $\mathcal{C}$ at $u \in \mathcal{C}$ is defined by

$$
\mathrm{N}_{\mathcal{C}}(\mathrm{u})=\{z \in \mathcal{H}:\langle z, y-u\rangle \leqslant 0, \forall y \in \mathcal{C}\} .
$$

Let $\varphi: \mathcal{C} \rightarrow \mathbb{R}$ is convex function. The sub-differential of $\varphi$ at $u \in \mathcal{C}$ is defined by

$$
\partial \varphi(\mathfrak{u})=\{z \in \mathcal{H}: \varphi(\mathrm{y})-\varphi(\mathfrak{u}) \geqslant\langle z, y-u\rangle, \forall y \in \mathcal{C}\} .
$$

Lemma 2.1 ([37, Theorem 27.4]). Let $\varphi: \mathcal{C} \rightarrow \mathcal{R}$ be a proper convex, subdifferentiable and lower semi-continuous function on $\mathcal{C}$. An element $\mathrm{u} \in \mathcal{C}$ is a minimizer of a function $\varphi$ iff

$$
0 \in \partial \varphi(u)+N_{\mathcal{C}}(u),
$$

where $\partial \varphi(u)$ stands for the sub-differential of $\varphi$ at $u \in \mathcal{C}$ and $\mathrm{N}_{\mathcal{C}}(\mathrm{u})$ the normal cone of $\mathcal{C}$ at $\mathrm{u}$. 
The metric projection $\mathrm{P}_{\mathcal{C}}(\mathrm{u})$ of $u \in \mathcal{H}$ onto a closed and convex subset $\mathcal{C}$ of $\mathcal{H}$ is defined by

$$
\mathrm{P}_{\mathcal{C}}(u)=\underset{y \in \mathcal{C}}{\arg \min }\|y-u\| .
$$

Lemma 2.2 ([4]). Assume that $\mathrm{P}_{\mathcal{C}}: \mathcal{H} \rightarrow \mathcal{C}$ is a metric projection such that

$$
\left\|y_{1}-P_{\mathcal{C}}\left(y_{2}\right)\right\|^{2}+\left\|P_{\mathcal{C}}\left(y_{2}\right)-y_{2}\right\|^{2} \leqslant\left\|y_{1}-y_{2}\right\|^{2}, y_{1} \in \mathcal{C}, y_{2} \in \mathcal{H} .
$$

(ii) $y_{3}=P_{\mathcal{C}}\left(y_{1}\right)$ if and only if

$$
\left\langle y_{1}-y_{3}, y_{2}-y_{3}\right\rangle \leqslant 0, \forall y_{2} \in \mathcal{C}
$$

$$
\left\|y_{1}-P_{\mathcal{C}}\left(y_{1}\right)\right\| \leqslant\left\|y_{1}-y_{2}\right\|, y_{2} \in \mathcal{C}, y_{1} \in \mathcal{H} .
$$

Lemma 2.3 ([41]). Assume that $\left\{q_{n}\right\} \subset(0,+\infty)$ is a sequence satisfying the following inequality

$$
\mathrm{q}_{\mathrm{n}+1} \leqslant\left(1-\gamma_{\mathrm{n}}\right) \mathrm{q}_{\mathrm{n}}+\gamma_{\mathrm{n}} \delta_{\mathrm{n}}, \forall \mathrm{n} \in \mathbb{N},
$$

where $\left\{\gamma_{\mathrm{n}}\right\} \subset(0,1)$ and $\left\{\delta_{\mathrm{n}}\right\} \subset \mathbb{R}$ satisfies the following conditions:

$$
\lim _{n \rightarrow \infty} \gamma_{n}=0, \sum_{n=1}^{+\infty} \gamma_{n}=+\infty, \text { and } \limsup _{n \rightarrow \infty} \delta_{n} \leqslant 0 \text {. }
$$

Then, $\lim _{n \rightarrow \infty} \mathrm{q}_{\mathrm{n}}=0$.

Lemma 2.4 ([26]). Assume that a sequence $\left\{\mathbf{q}_{n}\right\} \subset \mathbb{R}$ and there exists a subsequence $\left\{n_{i}\right\}$ of $\{n\}$ such that $q_{n_{i}}<$ $\mathrm{q}_{\mathrm{n}_{\mathrm{i}+1}}$, for all $i \in \mathbb{N}$. Then, there is a non decreasing sequence $\mathrm{m}_{\mathrm{k}} \subset \mathbb{N}$ such that $\mathrm{m}_{\mathrm{k}} \rightarrow \infty$ as $\mathrm{k} \rightarrow \infty$, and the following conditions are fulfilled by all (sufficiently large) numbers $k \in \mathbb{N}$ :

$$
\mathrm{q}_{\mathrm{m}_{\mathrm{k}}} \leqslant \mathrm{q}_{\mathrm{m}_{\mathrm{k}+1}} \text { and } \mathrm{q}_{\mathrm{k}} \leqslant \mathrm{q}_{\mathrm{m}_{\mathrm{k}+1}} .
$$

In fact, $m_{k}=\max \left\{j \leqslant k: q_{j} \leqslant v_{j+1}\right\}$.

Lemma 2.5 ([4]). For each $\mathrm{y}_{1}, \mathrm{y}_{2} \in \mathcal{H}$ and $\delta \in \mathbb{R}$, then the following relationships hold.

(i)

(ii)

$$
\left\|\delta y_{1}+(1-\delta) y_{2}\right\|^{2}=\delta\left\|y_{1}\right\|^{2}+(1-\delta)\left\|y_{2}\right\|^{2}-\delta(1-\delta)\left\|y_{1}-y_{2}\right\|^{2}
$$

$$
\left\|y_{1}+y_{2}\right\|^{2} \leqslant\left\|y_{1}\right\|^{2}+2\left\langle y_{2}, y_{1}+y_{2}\right\rangle
$$

In this article, the equilibrium problem is studied based on the following hypothesis.

(Ф1) pseudo-monotone on $\mathcal{C}$ if

$$
f\left(y_{1}, y_{2}\right) \geqslant 0 \Longrightarrow f\left(y_{2}, y_{1}\right) \leqslant 0, \forall y_{1}, y_{2} \in \mathcal{C} .
$$

(Ф2) Lipschitz-type continuous [27] on $\mathcal{C}$ if two constants $c_{1}, c_{2}>0$ such as

$$
f\left(y_{1}, y_{3}\right) \leqslant f\left(y_{1}, y_{2}\right)+f\left(y_{2}, y_{3}\right)+c_{1}\left\|y_{1}-y_{2}\right\|^{2}+c_{2}\left\|y_{2}-y_{3}\right\|^{2}, \forall y_{1}, y_{2}, y_{3} \in \mathcal{C} .
$$

(Ф3) $\limsup f\left(u_{n}, y\right) \leqslant f\left(p^{*}, y\right)$ for all $y \in \mathcal{C}$ and $\left\{u_{n}\right\} \subset \mathcal{C}$ satisfies $u_{n} \rightarrow p^{*}$;

(Ф4) $f(u, \cdot)$ is sub-differentiable and convex upon $\mathcal{H}$ for every each $u \in \mathcal{H}$.

\section{Main results}

In this section, we provide an iterative scheme for solving pseudo-monotone equilibrium problems that are based on Tran et al. [39] and Halpern [14]. The main algorithm has been given as Algorithm 1. 
Algorithm 1 (Halpern-type algorithm for pseudo-monotone equilibrium problems)

STEP 0: Choose $\mathfrak{u}_{0} \in \mathcal{C}, \zeta_{0}>0$ and a sequence $\alpha_{n} \subset(0,1)$ meet the following conditions, i.e.,

$$
\lim _{n \rightarrow \infty} \alpha_{n}=0 \text { and } \sum_{n=1}^{+\infty} \alpha_{n}=+\infty
$$

STEP 1: Compute

$$
y_{n}=\underset{y \in \mathcal{C}}{\arg \min }\left\{\zeta_{n} f\left(u_{n}, y\right)+\frac{1}{2}\left\|u_{n}-y\right\|^{2}\right\}
$$

If $u_{n}=y_{n}$, then stop the sequence. Otherwise, go to STEP 2 .

STEP 2: Construct a half-space

$$
\mathcal{H}_{n}=\left\{z \in \mathcal{H}:\left\langle u_{n}-\zeta_{n} \omega_{n}-y_{n}, z-y_{n}\right\rangle \leqslant 0\right\},
$$

where $\omega_{n} \in \partial_{2} f\left(u_{n}, y_{n}\right)$ satisfying $u_{n}-\zeta_{n} \omega_{n}-y_{n} \in N_{\mathcal{C}}\left(y_{n}\right)$. Compute

$$
t_{n}=\underset{y \in \mathcal{H}_{n}}{\arg \min }\left\{\zeta_{n} f\left(y_{n}, y\right)+\frac{1}{2}\left\|u_{n}-y\right\|^{2}\right\}
$$

STEP 3: Compute

$$
u_{n+1}=\alpha_{n} u_{0}+\left(1-\alpha_{n}\right) t_{n}
$$

STEP 4: Evaluate

$$
\zeta_{n+1}= \begin{cases}\min \left\{\zeta_{n}, \frac{\mu\left(\left\|u_{n}-y_{n}\right\|^{2}+\left\|t_{n}-y_{n}\right\|^{2}\right)}{2\left[f\left(u_{n}, t_{n}\right)-f\left(u_{n}, y_{n}\right)-f\left(y_{n}, t_{n}\right)\right]}\right\}, & \text { if } f\left(u_{n}, t_{n}\right)-f\left(u_{n}, y_{n}\right)-f\left(y_{n}, t_{n}\right)>0, \\ \zeta_{0}, & \text { otherwise. }\end{cases}
$$

Set $n:=n+1$ and go back to STEP 1 .

Remark 3.1. It can be easily prove that $\mathcal{C} \subset \mathcal{H}_{n}$. By $\mathrm{y}_{\mathrm{n}}$ and Lemma 2.1 , we have

$$
0 \in \partial_{2}\left\{\zeta_{n} f\left(u_{n}, y\right)+\frac{1}{2}\left\|u_{n}-y\right\|^{2}\right\}\left(y_{n}\right)+N_{\mathcal{C}}\left(y_{n}\right) .
$$

Indeed, for some $\omega_{n} \in \partial f\left(u_{n}, y_{n}\right)$ there exists $\bar{\omega}_{n} \in N_{\mathcal{C}}\left(y_{n}\right)$ such that

$$
\zeta_{n} \omega_{n}+y_{n}-u_{n}+\bar{\omega}_{n}=0 .
$$

Thus, we have

$$
\left\langle u_{n}-y_{n}, y-y_{n}\right\rangle=\zeta_{n}\left\langle\omega_{n}, y-y_{n}\right\rangle+\left\langle\bar{\omega}_{n}, y-y_{n}\right\rangle, \forall y \in \mathcal{C} .
$$

Due to $\bar{\omega}_{n} \in N_{\mathcal{C}}\left(y_{n}\right)$ means that $\left\langle\bar{\omega}_{n}, y-y_{n}\right\rangle \leqslant 0$, for all $y \in \mathcal{C}$. It implies that

$$
\left\langle u_{n}-y_{n}, y-y_{n}\right\rangle \leqslant \zeta_{n}\left\langle\omega_{n}, y-y_{n}\right\rangle, \forall y \in \mathcal{C},
$$

which implies that

$$
\left\langle u_{n}-\zeta_{n} \omega_{n}-y_{n}, y-y_{n}\right\rangle \leqslant 0, \forall y \in \mathcal{C} .
$$

It proves that $\mathcal{C} \subset \mathcal{H}_{n}$ for each $n \in \mathbb{N}$.

Theorem 3.2. Assume that $\left\{u_{n}\right\}$ is a sequence generated by Algorithm 1 and $u^{*} \in \beth_{E p}$. Then, $\left\{u_{n}\right\}$ converges strongly to $\mathrm{u}^{*}=\mathrm{P}_{\beth_{\mathrm{Ep}}}\left(\mathrm{u}_{0}\right)$. 
Proof. By the use of definition of $t_{n}$, we have

$$
0 \in \partial_{2}\left\{\zeta_{n} f\left(y_{n}, y\right)+\frac{1}{2}\left\|u_{n}-y\right\|^{2}\right\}\left(t_{n}\right)+N_{\mathcal{H}_{n}}\left(t_{n}\right)
$$

For some $\omega \in \partial f\left(y_{n}, t_{n}\right)$ there exists $\bar{\omega} \in N_{\mathcal{H}_{n}}\left(t_{n}\right)$ such that

$$
\zeta_{n} \omega+t_{n}-u_{n}+\bar{\omega}=0 .
$$

It follows that

$$
\left\langle u_{n}-t_{n}, y-t_{n}\right\rangle=\zeta_{n}\left\langle\omega, y-t_{n}\right\rangle+\left\langle\bar{\omega}, y-t_{n}\right\rangle, \forall y \in \mathcal{H}_{n} .
$$

Due to $\bar{\omega} \in N_{\mathcal{H}_{n}}\left(t_{n}\right)$ follows that $\left\langle\bar{\omega}, y-t_{n}\right\rangle \leqslant 0$, for all $y \in \mathcal{H}_{n}$. Thus, we have

$$
\left\langle u_{n}-t_{n}, y-t_{n}\right\rangle \leqslant \zeta_{n}\left\langle\omega, y-t_{n}\right\rangle, \forall y \in \mathcal{H}_{n} .
$$

Thus, $\omega \in \partial f\left(y_{n}, t_{n}\right)$ implies that

$$
f\left(y_{n}, y\right)-f\left(y_{n}, t_{n}\right) \geqslant\left\langle\omega, y-t_{n}\right\rangle, \forall y \in \mathcal{H} .
$$

From (3.1) and (3.2), we get

$$
\zeta_{n} f\left(y_{n}, y\right)-\zeta_{n} f\left(y_{n}, t_{n}\right) \geqslant\left\langle u_{n}-t_{n}, y-t_{n}\right\rangle, \forall y \in \mathcal{H}_{n} .
$$

By the use of definition of $\mathcal{H}_{n}$, we obtain

$$
\zeta_{n}\left\langle w_{n}, t_{n}-y_{n}\right\rangle \geqslant\left\langle u_{n}-y_{n}, t_{n}-y_{n}\right\rangle
$$

For $\omega_{n} \in \partial f\left(u_{n}, y_{n}\right)$, we get

$$
f\left(u_{n}, y\right)-f\left(u_{n}, y_{n}\right) \geqslant\left\langle\omega_{n}, y-y_{n}\right\rangle, \forall y \in \mathcal{H} .
$$

By taking $y=t_{n}$, we obtain

$$
f\left(u_{n}, t_{n}\right)-f\left(u_{n}, y_{n}\right) \geqslant\left\langle w_{n}, t_{n}-y_{n}\right\rangle, \forall y \in \mathcal{H}
$$

By the use of (3.4) and (3.5), we get

$$
\zeta_{n}\left\{f\left(u_{n}, t_{n}\right)-f\left(u_{n}, y_{n}\right)\right\} \geqslant\left\langle u_{n}-y_{n}, t_{n}-y_{n}\right\rangle .
$$

By substituting $y=u^{*}$ in (3.3), we get

$$
\zeta_{n} f\left(y_{n}, u^{*}\right)-\zeta_{n} f\left(y_{n}, t_{n}\right) \geqslant\left\langle u_{n}-t_{n}, u^{*}-t_{n}\right\rangle .
$$

Since $u^{*} \in \beth_{E p}$, we have $f\left(u^{*}, y_{n}\right) \geqslant 0$. From the pseudo-monotonicity of bi-function $f$ we get $f\left(y_{n}, u^{*}\right) \leqslant$ 0 . Thus, it gives that

$$
\left\langle u_{n}-t_{n}, t_{n}-u^{*}\right\rangle \geqslant \zeta_{n} f\left(y_{n}, t_{n}\right) .
$$

From the explanation $\zeta_{n+1}$, we obtain

$$
f\left(u_{n}, t_{n}\right)-f\left(u_{n}, y_{n}\right)-f\left(y_{n}, t_{n}\right) \leqslant \frac{\mu\left\|u_{n}-y_{n}\right\|^{2}+\mu\left\|t_{n}-y_{n}\right\|^{2}}{2 \zeta_{n+1}} .
$$

From (3.7) and (3.8), we obtain

$$
\left\langle u_{n}-t_{n}, t_{n}-u^{*}\right\rangle \geqslant \zeta_{n}\left\{f\left(u_{n}, t_{n}\right)-f\left(u_{n}, y_{n}\right)\right\}-\frac{\mu \zeta_{n}}{2 \zeta_{n+1}}\left\|u_{n}-y_{n}\right\|^{2}-\frac{\mu \zeta_{n}}{2 \zeta_{n+1}}\left\|t_{n}-y_{n}\right\|^{2} .
$$


From (3.6) and (3.9), we obtain

$$
\left\langle u_{n}-t_{n}, t_{n}-u^{*}\right\rangle \geqslant\left\langle u_{n}-y_{n}, t_{n}-y_{n}\right\rangle-\frac{\mu \zeta_{n}}{2 \zeta_{n+1}}\left\|u_{n}-y_{n}\right\|^{2}-\frac{\mu \zeta_{n}}{2 \zeta_{n+1}}\left\|t_{n}-y_{n}\right\|^{2} .
$$

We have the following formulas:

$$
\begin{aligned}
-2\left\langle u_{n}-t_{n}, t_{n}-u^{*}\right\rangle & =-\left\|u_{n}-u^{*}\right\|^{2}+\left\|t_{n}-u_{n}\right\|^{2}+\left\|t_{n}-u^{*}\right\|^{2}, \\
2\left\langle y_{n}-u_{n}, y_{n}-t_{n}\right\rangle & =\left\|u_{n}-y_{n}\right\|^{2}+\left\|t_{n}-y_{n}\right\|^{2}-\left\|u_{n}-t_{n}\right\|^{2} .
\end{aligned}
$$

Combining (3.10) and (3.11), we obtain

$$
\left\|t_{n}-u^{*}\right\|^{2} \leqslant\left\|u_{n}-u^{*}\right\|^{2}-\left(1-\frac{\mu \zeta_{n}}{\zeta_{n+1}}\right)\left\|u_{n}-y_{n}\right\|^{2}-\left(1-\frac{\mu \zeta_{n}}{\zeta_{n+1}}\right)\left\|t_{n}-y_{n}\right\|^{2}
$$

Due to $\zeta_{n} \rightarrow \zeta$, there exits a number $\epsilon \in(0,1-\mu)$ such that

$$
\lim _{n \rightarrow \infty}\left(1-\frac{\mu \zeta_{n}}{\zeta_{n+1}}\right)=1-\mu>\epsilon>0
$$

Thus, there exits a finite number $n_{1} \in \mathbb{N}$ such that

$$
\left(1-\frac{\mu \zeta_{n}}{\zeta_{n+1}}\right)>\epsilon>0, \forall n \geqslant n_{1}
$$

From (3.12), we obtain

$$
\left\|t_{n}-u^{*}\right\|^{2} \leqslant\left\|u_{n}-u^{*}\right\|^{2}, \forall n \geqslant n_{1} .
$$

Due to $u^{*} \in \beth_{\text {Ep }}$ and by the use of definition of $\left\{u_{n+1}\right\}$, we obtain

$$
\begin{aligned}
\left\|u_{n+1}-u^{*}\right\| & =\left\|\alpha_{n} u_{0}+\left(1-\alpha_{n}\right) t_{n}-u^{*}\right\| \\
& =\left\|\alpha_{n}\left[u_{0}-u^{*}\right]+\left(1-\alpha_{n}\right)\left[t_{n}-u^{*}\right]\right\| \leqslant \alpha_{n}\left\|u_{0}-u^{*}\right\|+\left(1-\alpha_{n}\right)\left\|t_{n}-u^{*}\right\| .
\end{aligned}
$$

Combining (3.13) and (3.14) and $\alpha_{n} \subset(0,1)$, we have

$$
\begin{aligned}
\left\|u_{n+1}-u^{*}\right\| & \leqslant \alpha_{n}\left\|u_{0}-u^{*}\right\|+\left(1-\alpha_{n}\right)\left\|u_{n}-u^{*}\right\| . \\
& \leqslant \max \left\{\left\|u_{0}-u^{*}\right\|,\left\|u_{n}-u^{*}\right\|\right\} \leqslant \max \left\{\left\|u_{0}-u^{*}\right\|,\left\|u_{n_{1}}-u^{*}\right\|\right\} .
\end{aligned}
$$

Thus, we conclude that the $\left\{u_{n}\right\}$ is bounded sequence. Next, we explain the strong convergence of the iterative sequence $\left\{u_{n}\right\}$ constructed by Algorithm 1. The Lipschitz-continuity and pseudo-monotone property of the bi-function $f$ indicates that the solution set $\beth_{E p}$ is a convex and closed set (see $[15,39]$ ). Let $u^{*}=P_{\beth_{\text {Ep }}}\left(u_{0}\right)$ and by Lemma 2.2 (ii), we have

$$
\left\langle u_{0}-u^{*}, y-u^{*}\right\rangle \leqslant 0, \forall y \in \beth_{E p} .
$$

Due to Lemma 2.5 (i) and (3.12), we get

$$
\begin{aligned}
\left\|u_{n+1}-u^{*}\right\|^{2}= & \left\|\alpha_{n} u_{0}+\left(1-\alpha_{n}\right) t_{n}-u^{*}\right\|^{2} \\
= & \left\|\alpha_{n}\left[u_{0}-u^{*}\right]+\left(1-\alpha_{n}\right)\left[t_{n}-u^{*}\right]\right\|^{2} \\
= & \alpha_{n}\left\|u_{0}-u^{*}\right\|^{2}+\left(1-\alpha_{n}\right)\left\|t_{n}-u^{*}\right\|^{2}-\alpha_{n}\left(1-\alpha_{n}\right)\left\|u_{0}-t_{n}\right\|^{2} \\
\leqslant & \alpha_{n}\left\|u_{0}-u^{*}\right\|^{2}+\left(1-\alpha_{n}\right)\left[\left\|u_{n}-u^{*}\right\|^{2}-\left(1-\frac{\mu \zeta_{n}}{\zeta_{n+1}}\right)\left\|u_{n}-y_{n}\right\|^{2}\right. \\
& \left.-\left(1-\frac{\mu \zeta_{n}}{\zeta_{n+1}}\right)\left\|t_{n}-y_{n}\right\|^{2}\right]-\alpha_{n}\left(1-\alpha_{n}\right)\left\|u_{0}-t_{n}\right\|^{2} \\
\leqslant & \alpha_{n}\left\|u_{0}-u^{*}\right\|^{2}+\left\|u_{n}-u^{*}\right\|^{2} \\
& -\left(1-\alpha_{n}\right)\left(1-\frac{\mu \zeta_{n}}{\zeta_{n+1}}\right)\left\|u_{n}-y_{n}\right\|^{2}-\left(1-\alpha_{n}\right)\left(1-\frac{\mu \zeta_{n}}{\zeta_{n+1}}\right)\left\|t_{n}-y_{n}\right\|^{2} .
\end{aligned}
$$


The above relation implies that

$$
\begin{gathered}
\left(1-\alpha_{n}\right)\left(1-\frac{\mu \zeta_{n}}{\zeta_{n+1}}\right)\left\|u_{n}-y_{n}\right\|^{2}+\left(1-\alpha_{n}\right)\left(1-\frac{\mu \zeta_{n}}{\zeta_{n+1}}\right)\left\|t_{n}-y_{n}\right\|^{2} \\
\leqslant \alpha_{n}\left\|u_{0}-u^{*}\right\|^{2}+\left\|u_{n}-u^{*}\right\|^{2}-\left\|u_{n+1}-u^{*}\right\|^{2} .
\end{gathered}
$$

The remainder of the proof shall be split into the following two parts.

Case 1: Assume that there is a fixed number $n_{2} \in \mathbb{N}$ such that

$$
\left\|u_{n+1}-u^{*}\right\| \leqslant\left\|u_{n}-u^{*}\right\|, \forall n \geqslant n_{2} .
$$

Thus, above implies that $\lim _{n \rightarrow \infty}\left\|u_{n}-u^{*}\right\|$ exists and let $\lim _{n \rightarrow \infty}\left\|u_{n}-u^{*}\right\|=$. From (3.15), we get

$$
\begin{gathered}
\left(1-\alpha_{n}\right)\left(1-\frac{\mu \zeta_{n}}{\zeta_{n+1}}\right)\left\|u_{n}-y_{n}\right\|^{2}+\left(1-\alpha_{n}\right)\left(1-\frac{\mu \zeta_{n}}{\zeta_{n+1}}\right)\left\|t_{n}-y_{n}\right\|^{2} \\
\leqslant \alpha_{n}\left\|u_{0}-u^{*}\right\|^{2}+\left\|u_{n}-u^{*}\right\|^{2}-\left\|u_{n+1}-u^{*}\right\|^{2} .
\end{gathered}
$$

The existence of $\lim _{n \rightarrow \infty}\left\|u_{n}-u^{*}\right\|=l$ and $\alpha_{n} \rightarrow 0$, we can deduce that

$$
\lim _{n \rightarrow \infty}\left\|u_{n}-y_{n}\right\|=\lim _{n \rightarrow \infty}\left\|t_{n}-y_{n}\right\|=0
$$

It follows that

$$
\lim _{n \rightarrow \infty}\left\|u_{n}-t_{n}\right\| \leqslant \lim _{n \rightarrow \infty}\left\|u_{n}-y_{n}\right\|+\lim _{n \rightarrow \infty}\left\|y_{n}-t_{n}\right\|=0
$$

Furthermore, we obtain

$$
\begin{aligned}
\left\|u_{n+1}-u_{n}\right\| & =\left\|\alpha_{n} u_{0}+\left(1-\alpha_{n}\right) t_{n}-u_{n}\right\| \\
& =\left\|\alpha_{n}\left[u_{0}-u_{n}\right]+\left(1-\alpha_{n}\right)\left[t_{n}-u_{n}\right]\right\| \leqslant \alpha_{n}\left\|u_{0}-u_{n}\right\|+\left(1-\alpha_{n}\right)\left\|t_{n}-u_{n}\right\| .
\end{aligned}
$$

It follows that

$$
\lim _{n \rightarrow \infty}\left\|u_{n+1}-u_{n}\right\|=0 .
$$

Thus, the implies that the sequences $\left\{y_{n}\right\}$ and $\left\{t_{n}\right\}$ are bounded. Due to the reflexivity of $\mathcal{H}$ and the boundedness of $\left\{u_{n}\right\}$ guarantees that there is a subsequence $\left\{u_{n_{k}}\right\}$ such that $\left\{u_{n_{k}}\right\} \rightarrow \hat{u} \in \mathcal{H}$ as $k \rightarrow \infty$. Next, we need to prove that $\hat{u} \in \beth_{\mathrm{Ep}}$. Due to the inequality (3.3), the Lipschitz-like condition of $f$ and (3.6), we obtain

$$
\begin{aligned}
\zeta_{n_{k}} f\left(y_{n_{k}}, y\right) \geqslant & \zeta_{n_{k}} f\left(y_{n_{k}}, t_{n_{k}}\right)+\left\langle u_{n_{k}}-t_{n_{k}}, y-t_{n_{k}}\right\rangle \\
\geqslant & \zeta_{n_{k}} f\left(u_{n_{k}}, t_{n_{k}}\right)-\zeta_{n_{k}} f\left(u_{n_{k}}, y_{n_{k}}\right)-\frac{\mu \zeta_{n_{k}}}{2 \zeta_{n_{k}+1}}\left\|u_{n_{k}}-y_{n_{k}}\right\|^{2} \\
& -\frac{\mu \zeta_{n_{k}}}{2 \zeta_{n_{k}+1}}\left\|y_{n_{k}}-t_{n_{k}}\right\|^{2}+\left\langle u_{n_{k}}-t_{n_{k}}, y-t_{n_{k}}\right\rangle \\
\geqslant & \left\langle u_{n_{k}}-y_{n_{k}}, t_{n_{k}}-y_{n_{k}}\right\rangle-\frac{\mu \zeta_{n_{k}}}{2 \zeta_{n_{k}+1}}\left\|u_{n_{k}}-y_{n_{k}}\right\|^{2} \\
& -\frac{\mu \zeta_{n_{k}}}{2 \zeta_{n_{k}+1}}\left\|y_{n_{k}}-t_{n_{k}}\right\|^{2}+\left\langle u_{n_{k}}-t_{n_{k}}, y-t_{n_{k}}\right\rangle,
\end{aligned}
$$

where $y \in \mathcal{H}_{n}$. From (3.16) and (3.17) imply that right-hand side reaches to zero. From $\zeta_{n_{k}}>0$, the condition (Ф3) and $y_{n_{k}} \rightarrow \hat{u}$, we obtain

$$
0 \leqslant \limsup _{k \rightarrow \infty} f\left(y_{n_{k}}, y\right) \leqslant f(\hat{u}, y), \forall y \in \mathcal{H}_{n}
$$


It follows that $f(\hat{u}, y) \geqslant 0$, for all $y \in \mathbb{C}$, and hence $\hat{u} \in \beth_{\text {Ep }}$. Next, consider

$$
\limsup _{n \rightarrow \infty}\left\langle u_{0}-u^{*}, u_{n}-u^{*}\right\rangle=\limsup _{k \rightarrow \infty}\left\langle u_{0}-u^{*}, u_{n_{k}}-u^{*}\right\rangle=\left\langle u_{0}-u^{*}, \hat{u}-u^{*}\right\rangle \leqslant 0 .
$$

By the use of $\lim _{n \rightarrow \infty}\left\|u_{n+1}-u_{n}\right\|=0$, we may conclude that

$$
\limsup _{n \rightarrow \infty}\left\langle u_{0}-u^{*}, u_{n+1}-u^{*}\right\rangle \leqslant \limsup _{n \rightarrow \infty}\left\langle u_{0}-u^{*}, u_{n+1}-u_{n}\right\rangle+\limsup _{n \rightarrow \infty}\left\langle u_{0}-u^{*}, u_{n}-u^{*}\right\rangle \leqslant 0 .
$$

From Lemma 2.5 (ii) and (3.12), we have

$$
\begin{aligned}
\left\|u_{n+1}-u^{*}\right\|^{2} & =\left\|\alpha_{n} u_{0}+\left(1-\alpha_{n}\right) t_{n}-u^{*}\right\|^{2} \\
& =\left\|\alpha_{n}\left[u_{0}-u^{*}\right]+\left(1-\alpha_{n}\right)\left[t_{n}-u^{*}\right]\right\|^{2} \\
& \leqslant\left(1-\alpha_{n}\right)^{2}\left\|t_{n}-u^{*}\right\|^{2}+2 \alpha_{n}\left\langle u_{0}-u^{*},\left(1-\alpha_{n}\right)\left[t_{n}-u^{*}\right]+\alpha_{n}\left[u_{0}-u^{*}\right]\right\rangle \\
& =\left(1-\alpha_{n}\right)^{2}\left\|t_{n}-u^{*}\right\|^{2}+2 \alpha_{n}\left\langle u_{0}-u^{*}, u_{n+1}-u^{*}\right\rangle \\
& \leqslant\left(1-\alpha_{n}\right)\left\|u_{n}-u^{*}\right\|^{2}+2 \alpha_{n}\left\langle u_{0}-u^{*}, u_{n+1}-u^{*}\right\rangle .
\end{aligned}
$$

From (3.18), (3.19), and using Lemma 2.3, we may deduce that $\left\|u_{n}-u^{*}\right\| \rightarrow 0$ as $n \rightarrow \infty$.

Case 2: Assume there exits a subsequence $\left\{n_{i}\right\}$ of $\{n\}$ such that

$$
\left\|u_{n_{i}}-u^{*}\right\| \leqslant\left\|u_{n_{i+1}}-u^{*}\right\|, \forall i \in \mathbb{N} .
$$

Thus, by Lemma 2.4, there exists a sequence $\left\{m_{k}\right\} \subset \mathbb{N}$ as $\left\{m_{k}\right\} \rightarrow \infty$, such that

$$
\left\|u_{m_{k}}-u^{*}\right\| \leqslant\left\|u_{m_{k+1}}-u^{*}\right\| \text { and }\left\|u_{k}-u^{*}\right\| \leqslant\left\|u_{m_{k+1}}-u^{*}\right\| \text {, for all } k \in \mathbb{N} \text {. }
$$

Similar to Case 1, expression (3.15) provides that

$$
\begin{aligned}
& \left(1-\alpha_{m_{k}}\right)\left(1-\frac{\mu \zeta_{m_{k}}}{\zeta_{m_{k}+1}}\right)\left\|u_{m_{k}}-y_{m_{k}}\right\|^{2}+\left(1-\alpha_{m_{k}}\right)\left(1-\frac{\mu \zeta_{m_{k}}}{\zeta_{m_{k}+1}}\right)\left\|t_{m_{k}}-y_{m_{k}}\right\|^{2} \\
& \quad \leqslant \alpha_{m_{k}}\left\|u_{0}-u^{*}\right\|^{2}+\left\|u_{m_{k}}-u^{*}\right\|^{2}-\left\|u_{m_{k}+1}-u^{*}\right\|^{2}
\end{aligned}
$$

Due to $\alpha_{m_{k}} \rightarrow 0$, we deduce the following:

$$
\lim _{n \rightarrow \infty}\left\|u_{m_{k}}-y_{m_{k}}\right\|=\lim _{n \rightarrow \infty}\left\|t_{m_{k}}-y_{m_{k}}\right\|=0
$$

Also, we can obtain

$$
\begin{aligned}
\left\|u_{m_{k}+1}-u_{m_{k}}\right\| & =\left\|\alpha_{m_{k}} u_{0}+\left(1-\alpha_{m_{k}}\right) t_{m_{k}}-u_{m_{k}}\right\| \\
& =\left\|\alpha_{m_{k}}\left[u_{0}-u_{m_{k}}\right]+\left(1-\alpha_{m_{k}}\right)\left[t_{m_{k}}-u_{m_{k}}\right]\right\| \\
& \leqslant \alpha_{m_{k}}\left\|u_{0}-u_{m_{k}}\right\|+\left(1-\alpha_{m_{k}}\right)\left\|t_{m_{k}}-u_{m_{k}}\right\| \longrightarrow 0 .
\end{aligned}
$$

We use the same argument as in Case 1, which is as follows:

$$
\limsup _{k \rightarrow \infty}\left\langle u_{0}-u^{*}, u_{m_{k}+1}-u^{*}\right\rangle \leqslant 0 .
$$

Now, using expressions (3.19) and (3.20), we have

$$
\begin{aligned}
\left\|u_{m_{k}+1}-u^{*}\right\|^{2} & \leqslant\left(1-\alpha_{m_{k}}\right)\left\|u_{m_{k}}-u^{*}\right\|^{2}+2 \alpha_{m_{k}}\left\langle u_{0}-u^{*}, u_{m_{k}+1}-u^{*}\right\rangle \\
& \leqslant\left(1-\alpha_{m_{k}}\right)\left\|u_{m_{k}+1}-u^{*}\right\|^{2}+2 \alpha_{m_{k}}\left\langle u_{0}-u^{*}, u_{m_{k}+1}-u^{*}\right\rangle .
\end{aligned}
$$


It continues from that

$$
\left\|u_{m_{k}+1}-u^{*}\right\|^{2} \leqslant 2\left\langle u_{0}-u^{*}, u_{m_{k}+1}-u^{*}\right\rangle \text {. }
$$

Since $\alpha_{m_{k}} \rightarrow 0$ and $\left\|u_{m_{k}}-u^{*}\right\|$ is bounded, thus from (3.21) and (3.22) we obtain

$$
\left\|u_{m_{k}+1}-u^{*}\right\|^{2} \rightarrow 0, \text { as } k \rightarrow \infty .
$$

It implies that

$$
\lim _{n \rightarrow \infty}\left\|u_{k}-u^{*}\right\|^{2} \leqslant \lim _{n \rightarrow \infty}\left\|u_{m_{k}+1}-u^{*}\right\|^{2} \leqslant 0 .
$$

Consequently, $u_{n} \rightarrow u^{*}$. This completes the proof of the theorem.

\section{Applications}

Now, we consider the application of our main results to solve the problem of classic variational inequalities [38]. A mapping $\mathcal{M}: \mathcal{H} \rightarrow \mathcal{H}$ is defined as follows:

$$
\text { Find } u^{*} \in \mathcal{C} \text { such that }\left\langle\mathcal{M}\left(u^{*}\right), y-u^{*}\right\rangle \geqslant 0, \forall y \in \mathcal{C} \text {. }
$$

We consider the following conditions to study variational inequalities.

$(\mathcal{M} 1)$ The solution set of the problem (VIP) denoted by $\mathrm{VI}(\mathcal{M}, \mathcal{C})$ is non-empty.

$(\mathcal{N} 2) \mathcal{M}: \mathcal{H} \rightarrow \mathcal{H}$ is called to be a pseudo-monotone, i.e.,

$$
\langle\mathcal{M}(u), y-u\rangle \geqslant 0 \Longrightarrow\langle\mathcal{M}(y), u-y\rangle \leqslant 0, \forall u, y \in \mathcal{C} .
$$

( $\mathcal{M} 3) \mathcal{M}: \mathcal{H} \rightarrow \mathcal{H}$ is called to be a Lipschitz continuous if there exits a constants $\mathrm{L}>0$ such that

$$
\|\mathcal{M}(u)-\mathcal{M}(y)\| \leqslant L\|u-y\|, \forall u, y \in \mathcal{C} ;
$$

$(\mathcal{M} 4) \lim \sup \left\langle\mathcal{M}\left(u_{n}\right), y-u_{n}\right\rangle \leqslant\left\langle\mathcal{M}\left(q^{*}\right), y-q^{*}\right\rangle$ for every $y \in \mathcal{C}$ and $\left\{u_{n}\right\} \subset \mathcal{C}$ satisfies $u_{n} \rightarrow q^{*}$.

By the use of $f(u, y):=\langle\mathcal{M}(u), y-u\rangle$, for all $u, y \in \mathcal{C}$, thus, our main problem turns into the problem of variational inequalities outlined above while $L=2 c_{1}=2 c_{2}$. From the above value of the bi-function $f$, we get

$$
\left\{\begin{array}{l}
y_{n}=\underset{y \in \mathcal{C}}{\arg \min }\left\{\zeta_{n} f\left(u_{n}, y\right)+\frac{1}{2}\left\|u_{n}-y\right\|^{2}\right\}=P_{\mathcal{C}}\left(u_{n}-\zeta_{n} \mathcal{M}\left(u_{n}\right)\right), \\
t_{n}=\underset{y \in \mathcal{H}_{n}}{\arg \min }\left\{\zeta_{n} f\left(y_{n}, y\right)+\frac{1}{2}\left\|u_{n}-y\right\|^{2}\right\}=P_{\mathcal{H}_{n}}\left(u_{n}-\zeta_{n} \mathcal{M}\left(y_{n}\right)\right) .
\end{array}\right.
$$

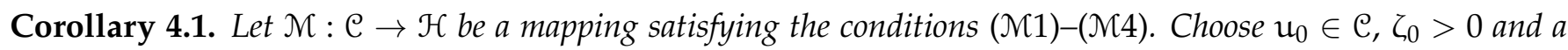
sequence $\alpha_{n} \subset(0,1)$ meet the following conditions, i.e.,

$$
\lim _{n \rightarrow \infty} \alpha_{n}=0 \text { and } \sum_{n=1}^{+\infty} \alpha_{n}=+\infty .
$$

Assume that $\left\{u_{n}\right\}$ generated as follows:

$$
\left\{\begin{array}{l}
y_{n}=P_{\mathcal{C}}\left(u_{n}-\zeta_{n} \mathcal{M}\left(u_{n}\right)\right) \\
t_{n}=P_{\mathcal{H}_{n}}\left(u_{n}-\zeta_{n} \mathcal{M}\left(y_{n}\right)\right) \\
u_{n+1}=\alpha_{n} u_{0}+\left(1-\alpha_{n}\right) t_{n}
\end{array}\right.
$$


where $\mathcal{H}_{n}=\left\{z \in \mathcal{H}:\left\langle u_{n}-\zeta_{n} \mathcal{N}\left(u_{n}\right)-y_{n}, z-y_{n}\right\rangle \leqslant 0\right\}$. The step size rule revised in the following way:

$$
\zeta_{n+1}= \begin{cases}\min \left\{\zeta_{n}, \frac{\mu\left\|u_{n}-y_{n}\right\|^{2}+\mu\left\|t_{n}-y_{n}\right\|^{2}}{2\left\langle\mathcal{M}\left(u_{n}\right)-\mathcal{M}\left(y_{n}\right), t_{n}-y_{n}\right\rangle}\right\}, & \text { if }\left\langle\mathcal{M}\left(u_{n}\right)-\mathcal{M}\left(y_{n}\right), t_{n}-y_{n}\right\rangle>0, \\ \zeta_{n}, & \text { else. }\end{cases}
$$

Then, the sequence $\left\{\mathrm{u}_{\mathrm{n}}\right\}$ converges strongly to $\mathrm{u}^{*} \in \mathrm{VI}(\mathcal{M}, \mathcal{C})$.

Next, we study the application of our main results to solve the fixed-point problems associated with the $\mathrm{k}$-strict pseudo-contraction mapping. The fixed point problem for a mapping $\mathcal{N}: \mathcal{H} \rightarrow \mathcal{H}$ is defined as follows:

$$
\text { Find } u^{*} \in \mathcal{C} \text { such that } \mathcal{N}\left(u^{*}\right)=u^{*} \text {. }
$$

We assume that the following conditions have been satisfied.

(N1) $\mathcal{N}: \mathcal{C} \rightarrow \mathcal{C}$ is said to be a $\mathrm{k}$-strict pseudo-contraction [8] on $\mathcal{C}$ if

$$
\|T u-T y\|^{2} \leqslant\|u-y\|^{2}+k\|(u-T u)-(y-T y)\|^{2}, \forall u, y \in \mathcal{C} ;
$$

(N2) $\limsup _{n \rightarrow \infty}\left\langle u_{n}-\mathcal{N}\left(u_{n}\right), y-u_{n}\right\rangle \leqslant\left\langle q^{*}-G\left(q^{*}\right), y-q^{*}\right\rangle$ for each $y \in \mathcal{C}$ and $\left\{u_{n}\right\} \subset \mathcal{C}$ satisfies $u_{n} \rightarrow q^{*}$.

If we consider that the mapping $\mathcal{N}$ is a $k$-strict pseudocontraction and weakly continuous then $f(u, y)=$ $\langle u-\mathcal{N u}, \mathrm{y}-\mathrm{u}\rangle$ satisfies the conditions $(\Phi 1)-(\Phi 4)$ and $2 \mathrm{c}_{1}=2 \mathrm{c}_{2}=\frac{3-2 \mathrm{k}}{1-\mathrm{k}}$.

$$
\left\{\begin{array}{l}
y_{n}=\underset{y \in \mathcal{C}}{\arg \min }\left\{\zeta_{n} f\left(u_{n}, y\right)+\frac{1}{2}\left\|u_{n}-y\right\|^{2}\right\}=P_{\mathcal{C}}\left[u_{n}-\zeta_{n}\left(u_{n}-\mathcal{N}\left(u_{n}\right)\right)\right] \\
t_{n}=\underset{y \in \mathcal{H}_{n}}{\arg \min }\left\{\zeta_{n} f\left(y_{n}, y\right)+\frac{1}{2}\left\|u_{n}-y\right\|^{2}\right\}=P_{\mathcal{H}_{n}}\left[u_{n}-\zeta_{n}\left(y_{n}-\mathcal{N}\left(y_{n}\right)\right)\right]
\end{array}\right.
$$

Corollary 4.2. Let $\mathcal{C}$ be a nonempty, convex and closed subset of a Hilbert space $\mathcal{H}$. Moreover, $\mathcal{N}: \mathcal{C} \rightarrow \mathcal{C}$ is a $\mathrm{k}$-strict pseudo-contraction and weakly continuous with solution set $\operatorname{Fix}(\mathcal{N}) \neq \emptyset$. Choose $\mathrm{u}_{0} \in \mathcal{C}, \zeta_{0}>0$ and a sequence $\alpha_{n} \subset(0,1)$ meet the following conditions, i.e.,

$$
\lim _{n \rightarrow \infty} \alpha_{n}=0 \text { and } \sum_{n}^{+\infty} \alpha_{n}=+\infty .
$$

Assume that $\left\{\mathfrak{u}_{\mathrm{n}}\right\}$ generated as follows:

$$
\left\{\begin{array}{l}
y_{n}=P_{\mathcal{C}}\left[u_{n}-\zeta_{n}\left(u_{n}-\mathcal{N}\left(u_{n}\right)\right)\right] \\
t_{n}=P_{\mathcal{H}_{n}}\left[u_{n}-\zeta_{n}\left(y_{n}-\mathcal{N}\left(y_{n}\right)\right)\right] \\
u_{n+1}=\alpha_{n} u_{0}+\left(1-\alpha_{n}\right) t_{n}
\end{array}\right.
$$

where $\mathcal{H}_{n}=\left\{z \in \mathcal{H}:\left\langle\left(1-\zeta_{n}\right) u_{n}+\zeta_{n} \mathcal{N}\left(u_{n}\right)-y_{n}, z-y_{n}\right\rangle \leqslant 0\right\}$. Compute $\zeta_{n+1}= \begin{cases}\min \left\{\zeta_{n}, \frac{\mu\left\|u_{n}-y_{n}\right\|^{2}+\mu\left\|t_{n}-y_{n}\right\|^{2}}{2\left\langle\left(u_{n}-y_{n}\right)-\left[\mathcal{N}\left(u_{n}\right)-\mathcal{N}\left(y_{n}\right)\right], t_{n}-y_{n}\right\rangle}\right\}, & \text { if }\left\langle\left(u_{n}-y_{n}\right)-\left[\mathcal{N}\left(u_{n}\right)-\mathcal{N}\left(y_{n}\right)\right], t_{n}-y_{n}\right\rangle>0 \\ \zeta_{n}, & \text { else. }\end{cases}$

Then, sequence $\left\{u_{n}\right\}$ strongly converges to $u^{*} \in \operatorname{Fix}(\mathcal{N})$. 


\section{Numerical illustrations}

The numerical results of the study are provided in the following section to illustrate the effectiveness of the proposed method. We studied one test problem in finite-dimensional space and second in-finite dimensional space. From the experiments, we have seen how the starting point affects the efficiency of algorithms.

Example 5.1. Assume that $f: \mathcal{C} \times \mathcal{C} \rightarrow \mathbb{R}$ is defined by

$$
f(u, y)=\langle P u+Q y+c, y-u\rangle, \forall u, y \in \mathcal{C},
$$

where $c \in \mathbb{R}^{n}$ and $P, Q$ are matrices of order $n$. The matrix $P$ is symmetric positive semi-definite and the matrix $Q-P$ is symmetric negative semi-definite with Lipschitz-type constants $c_{1}=c_{2}=\frac{1}{2}\|P-Q\|$ (see [39] for details). The matrices $P, Q$ and vector $c$ are defined as follows:

$$
\mathrm{P}=\left(\begin{array}{ccccc}
3.1 & 2 & 0 & 0 & 0 \\
2 & 3.6 & 0 & 0 & 0 \\
0 & 0 & 3.5 & 2 & 0 \\
0 & 0 & 2 & 3.3 & 0 \\
0 & 0 & 0 & 0 & 3
\end{array}\right), \quad \mathrm{Q}=\left(\begin{array}{ccccc}
1.6 & 1 & 0 & 0 & 0 \\
1 & 1.6 & 0 & 0 & 0 \\
0 & 0 & 1.5 & 1 & 0 \\
0 & 0 & 1 & 1.5 & 0 \\
0 & 0 & 0 & 0 & 2
\end{array}\right), \quad \mathrm{c}=\left(\begin{array}{c}
1 \\
-2 \\
-1 \\
2 \\
-1
\end{array}\right)
$$

The constraint set $C \subset \mathbb{R}^{n}$ is defined by

$$
C:=\left\{u \in \mathbb{R}^{n}:-10 \leqslant u_{i} \leqslant 10\right\} .
$$

The numerically and graphically findings of the three methods are shown in Figures 1-5 and Table 1 by using $y_{-1}=(0,0,0,0,0)^{\mathrm{T}}$ and letting different starting points $u_{0}=y_{0}$. The control parameters criteria are used as mentioned in the following:

(i) $\zeta=\frac{1}{5 c_{1}}, \alpha_{n}=\frac{1}{5(n+2)}$ and $D_{n}=\left\|u_{n}-y_{n}\right\|^{2}$ for Algorithm 2 (Algorithm-2) in [15].

(ii) $\zeta_{0}=0.22, \mu=0.33, \alpha_{n}=\frac{1}{(n+1)^{0.5}}, D_{n}=\left\|u_{n}-y_{n}\right\|^{2}$ for Algorithm 4.1 (Algorithm-4.1) in [22].

(iii) $\zeta_{0}=0.22, \mu=0.33, \alpha_{n}=\frac{1}{5(n+2)}$ and $D_{n}=\left\|u_{n}-y_{n}\right\|^{2}$ for Algorithm 1 (Algorithm-1).

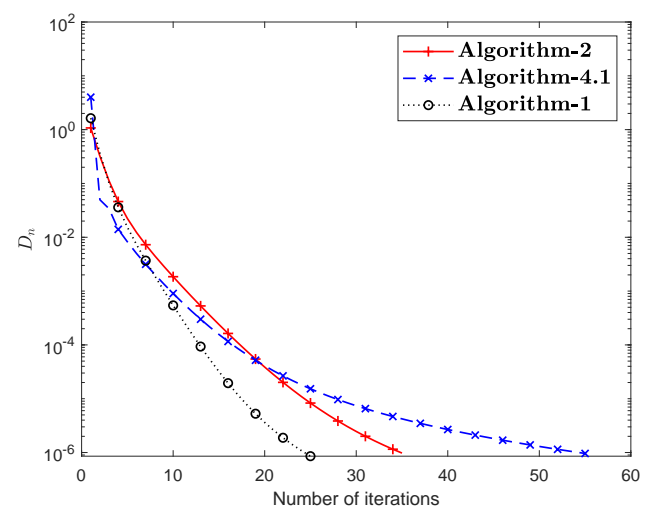

(a)

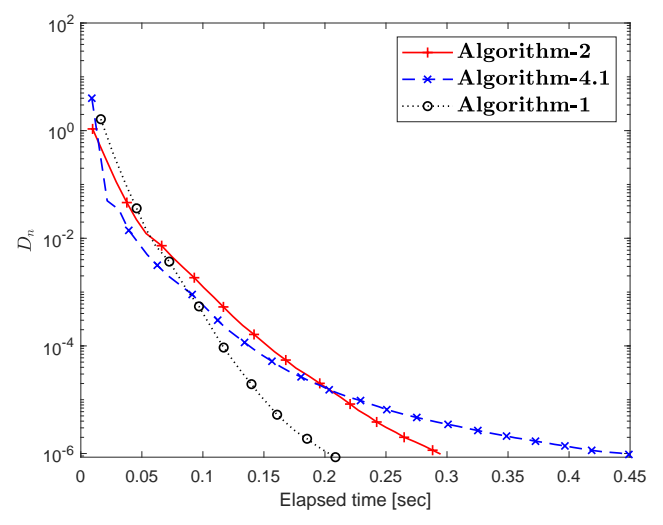

(b)

Figure 1: Numerical comparison behaviour of Algorithm 1 with Algorithm 4.1 in [22] and Algorithm 2 in [15] when $\mathfrak{u}_{0}=(1,1,1,1,1)^{\top}$. 


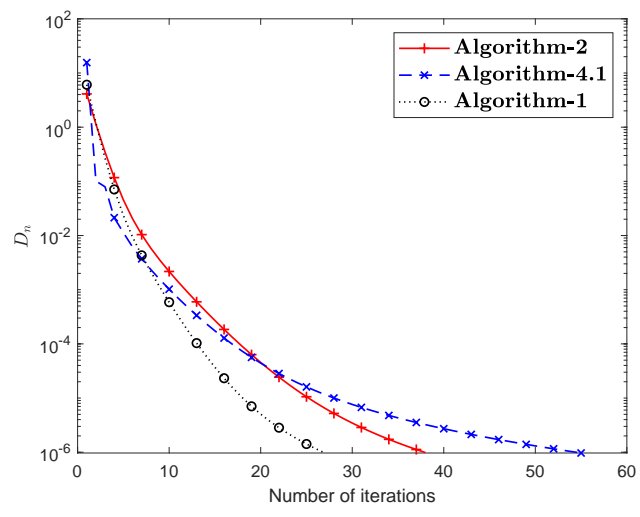

(a)

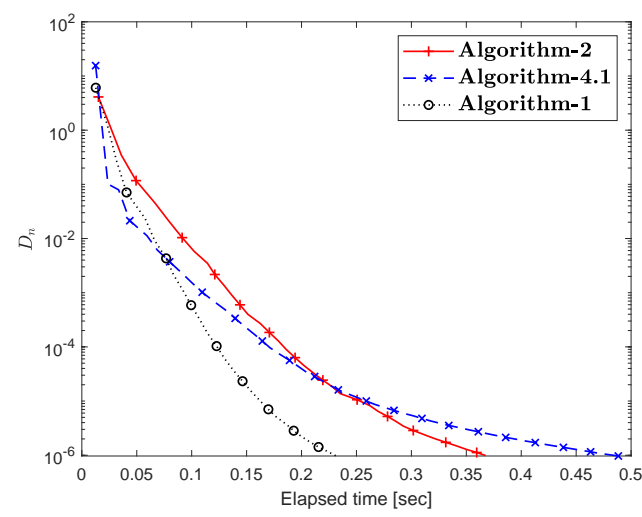

(b)

Figure 2: Numerical comparison behaviour of Algorithm 1 with Algorithm 4.1 in [22] and Algorithm 2 in [15] when $u_{0}=(2,2,2,2,2)^{\mathrm{T}}$.

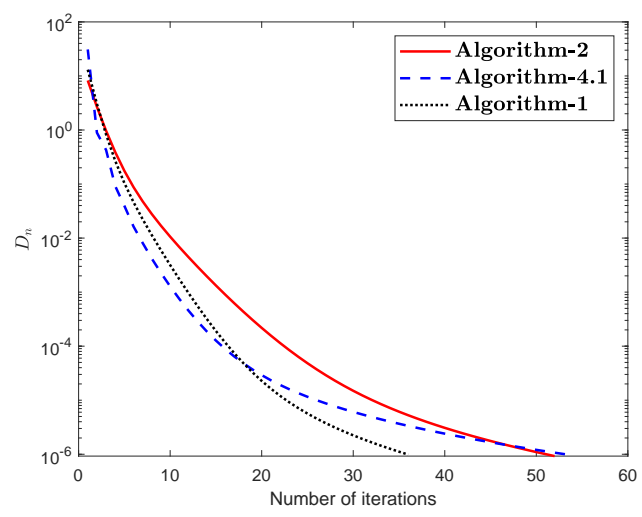

(a)

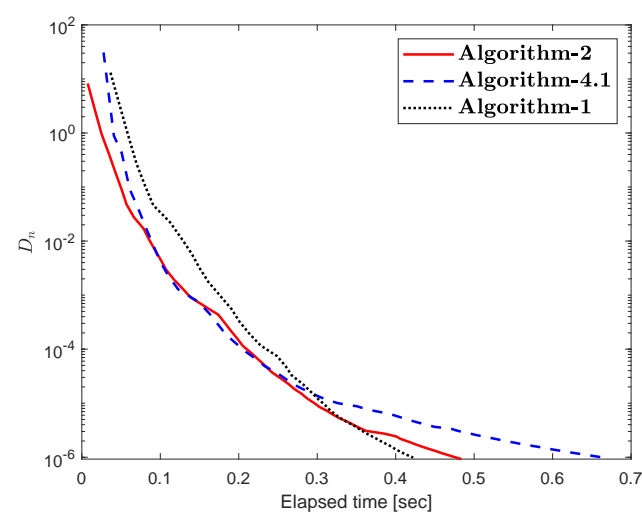

(b)

Figure 3: Numerical comparison behaviour of Algorithm 1 with Algorithm 4.1 in [22] and Algorithm 2 in [15] when $\mathrm{u}_{0}=(-1,-2,-4,-1,-5)^{\mathrm{T}}$.

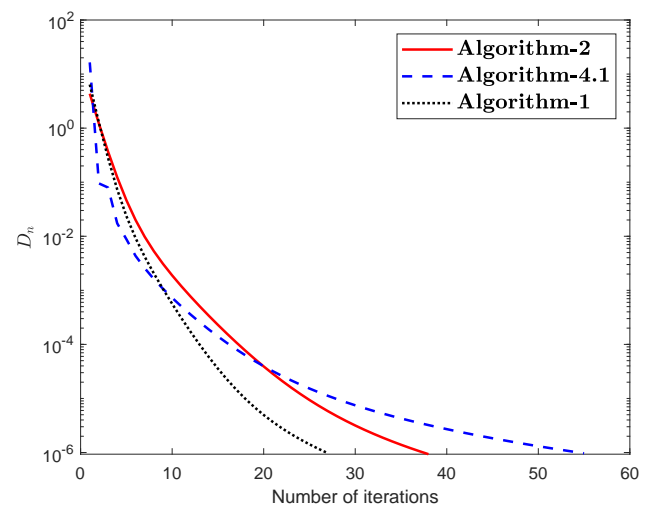

(a)

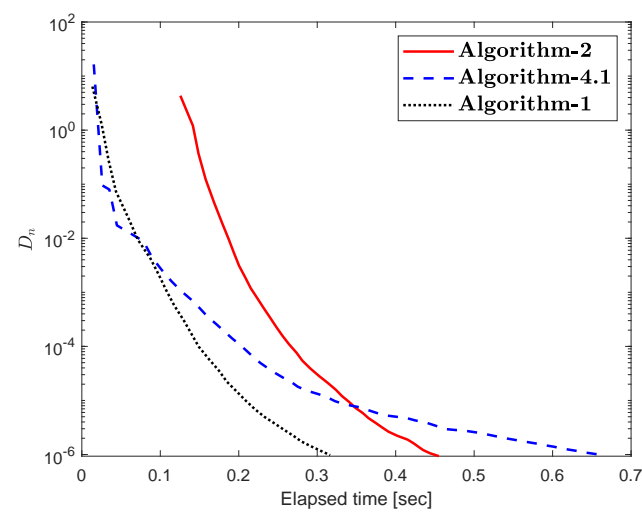

(b)

Figure 4: Numerical comparison behaviour of Algorithm 1 with Algorithm 4.1 in [22] and Algorithm 2 in [15] when $\mathfrak{u}_{0}=(-2,-2,-2,-2,-2)^{\top}$. 


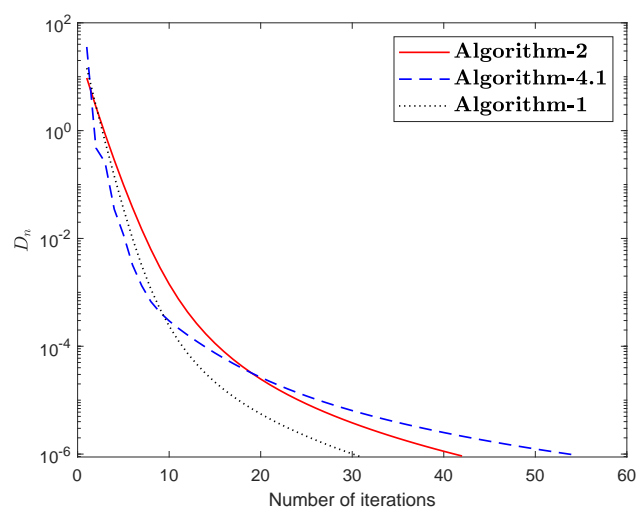

(a)

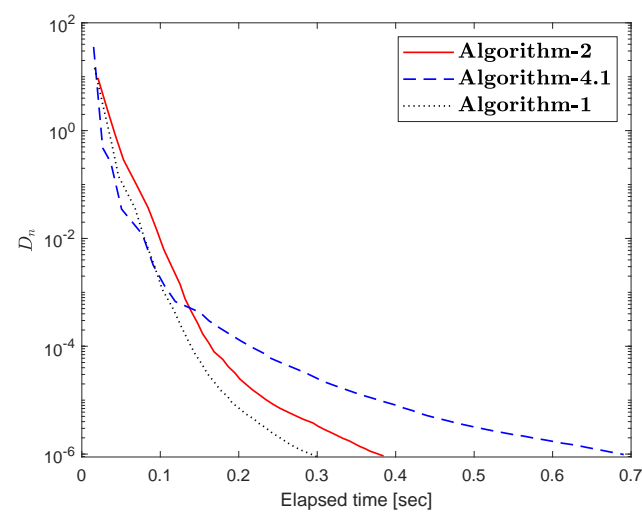

(b)

Figure 5: Numerical comparison behaviour of Algorithm 1 with Algorithm 4.1 in [22] and Algorithm 2 in [15] when $\mathfrak{u}_{0}=(1,2,-3,-4,5)^{\mathrm{T}}$.

Table 1: Numerical results data for Figures 1-5.

\begin{tabular}{cccc|ccc}
\hline & \multicolumn{3}{c}{ Number of iterations } & \multicolumn{3}{c}{ CPU time in seconds } \\
\hline$u_{0}$ & Algorithm-2 & Algorithm-4.1 & Algorithm-1 & Algorithm-2 & Algorithm-4.1 & Algorithm-1 \\
\hline$(1,1,1,1,1)^{\top}$ & 35 & 52 & 25 & 0.2947482 & 0.4185463 & 0.2085999 \\
$(2,2,2,2,2)^{\top}$ & 38 & 55 & 27 & 0.3676513 & 0.4883899 & 0.2315488 \\
$(-1,-2,-4,-1,-5)^{\top}$ & 52 & 54 & 36 & 0.4838859 & 0.6724367 & 0.4223327 \\
$(-2,-2,-2,-2,-2)^{\top}$ & 38 & 55 & 27 & 0.4550715 & 0.6656828 & 0.3164745 \\
$(1,2,-3,-4,5)^{\top}$ & 42 & 54 & 31 & 0.3845626 & 0.6906321 & 0.30042470 \\
\hline
\end{tabular}

Example 5.2. Suppose that $\mathcal{H}=\mathrm{L}^{2}([0,1])$ is a Hilbert space with an inner product

$$
\langle u, y\rangle=\int_{0}^{1} u(t) y(t) d t, \forall u, y \in \mathrm{L}^{2}([0,1]),
$$

where the induced norm is defined by

$$
\|\mathrm{u}\|=\sqrt{\int_{0}^{1} \mathrm{u}^{2}(\mathrm{t}) \mathrm{dt}}, \forall \mathrm{u} \in \mathrm{L}^{2}([0,1]) .
$$

Moreover, assume that a bi-function $\mathrm{f}: \mathcal{H} \times \mathcal{H} \rightarrow \mathcal{R}$ is defined by

$$
f(u, y)=\langle\mathcal{M}(u), y-u\rangle,
$$

where $\mathcal{M}(u(t))=\int_{0}^{t} u(s) d s$ for every $u \in L^{2}([0,1])$ and $t \in[0,1]$. The feasible set $\mathcal{C}:=\left\{u \in \mathrm{L}^{2}([0,1])\right.$ : $\left.\int_{0}^{1} t u(t) d t=2\right\}$. It is easy to note that that $f$ is monotone and Lipschitz-type continuous with $c_{1}=c_{2}=\frac{1}{\pi}$ (see [4]). The projection on set $\mathcal{C}$ is computed in the following way:

$$
P_{\mathcal{C}}(u)(t):=u(t)-\frac{\int_{0}^{1} t u(t) d t-2}{\int_{0}^{1} t^{2} d t} t, t \in[0,1] .
$$

The numerically and graphically findings of the three methods are shown in Figures 6-9 and Table 2 by using $y_{-1}=t$ and letting different starting points $u_{0}=y_{0}$. The control parameters criteria are used as mentioned in the following:

(i) $\zeta=\frac{1}{7 c_{1}}, \alpha_{n}=\frac{1}{10(n+2)}$ and $D_{n}=\left\|u_{n}-y_{n}\right\|^{2}$ for Algorithm 2 (Algorithm-2) in [15].

(ii) $\zeta_{0}=0.12, \mu=0.33, \alpha_{n}=\frac{1}{(n+1)^{0.5}}, D_{n}=\left\|u_{n}-y_{n}\right\|^{2}$ for Algorithm 4.1 (Algorithm-4.1) in [22]. 
(iii) $\zeta_{0}=0.12, \mu=0.33, \alpha_{n}=\frac{1}{10(n+2)}$ and $D_{n}=\left\|u_{n}-y_{n}\right\|^{2}$ for Algorithm 1 (Algorithm-1).

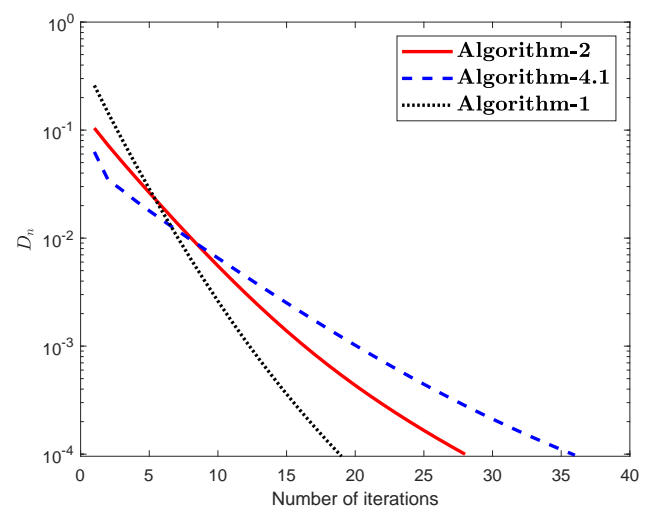

(a)

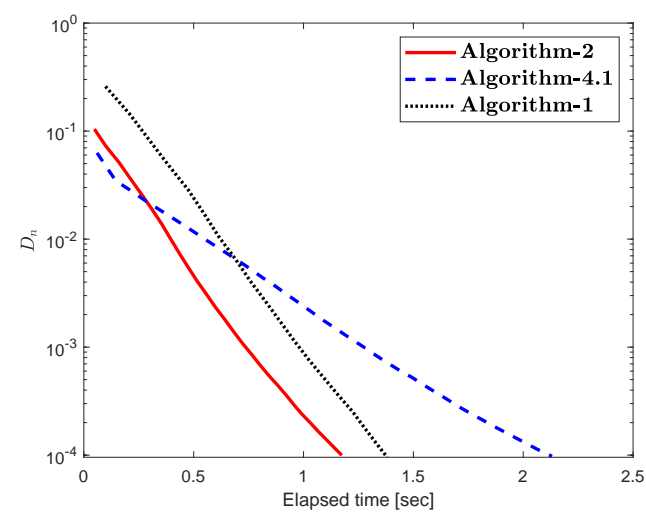

(b)

Figure 6: Numerical comparison behaviour of Algorithm 1 with Algorithm 4.1 in [22] and Algorithm 2 in [15] when $u_{0}=3 t^{2}$.

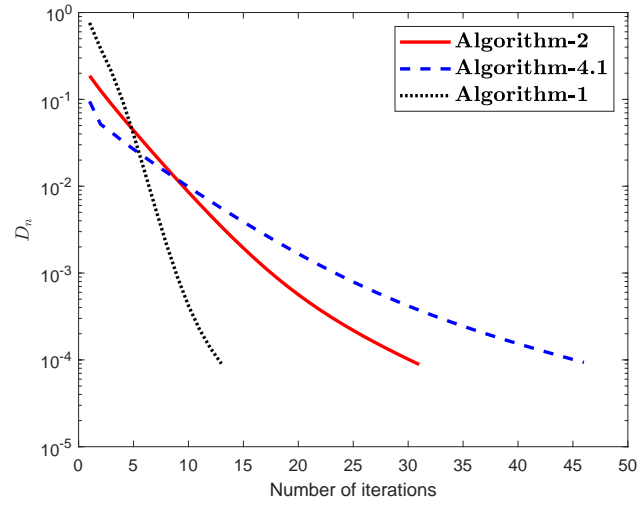

(a)

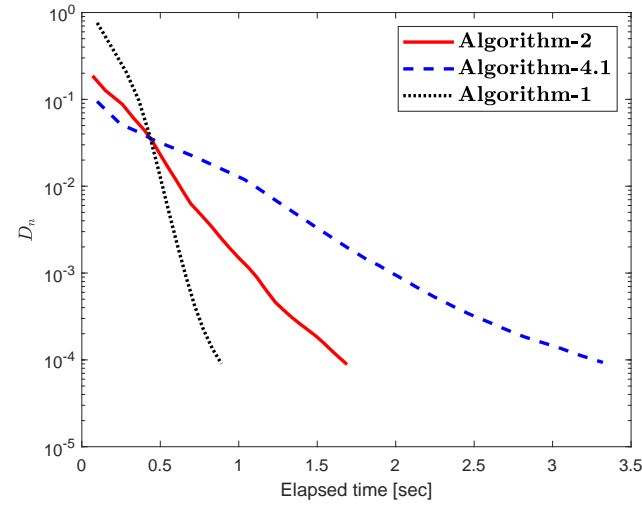

(b)

Figure 7: Numerical comparison behaviour of Algorithm 1 with Algorithm 4.1 in [22] and Algorithm 2 in [15] when $u_{0}=-2 t+5 t^{2}$.

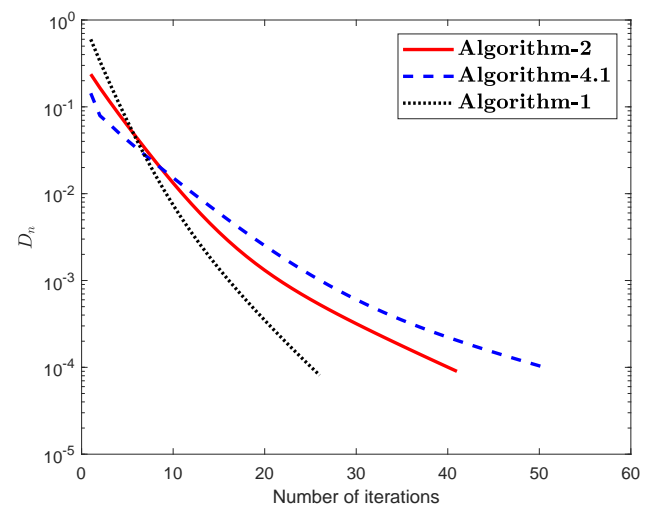

(a)

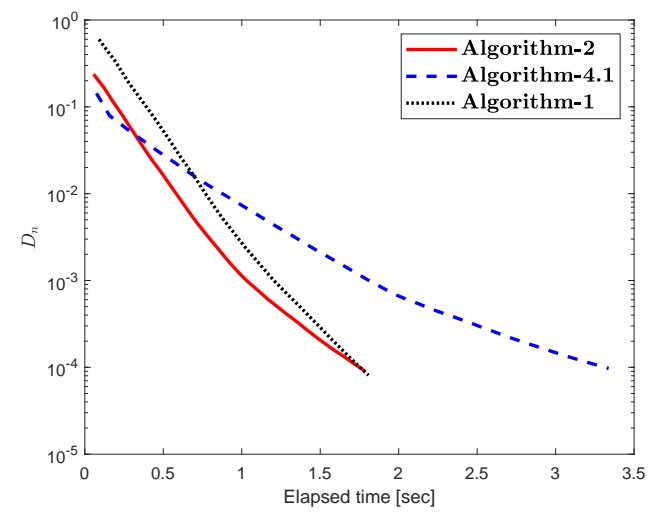

(b)

Figure 8: Numerical comparison behaviour of Algorithm 1 with Algorithm 4.1 in [22] and Algorithm 2 in [15] when $u_{0}=2 e^{t} \sin (t)$. 


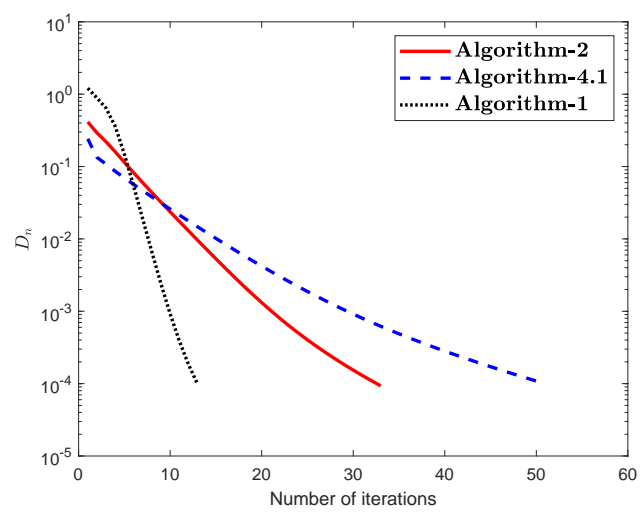

(a)

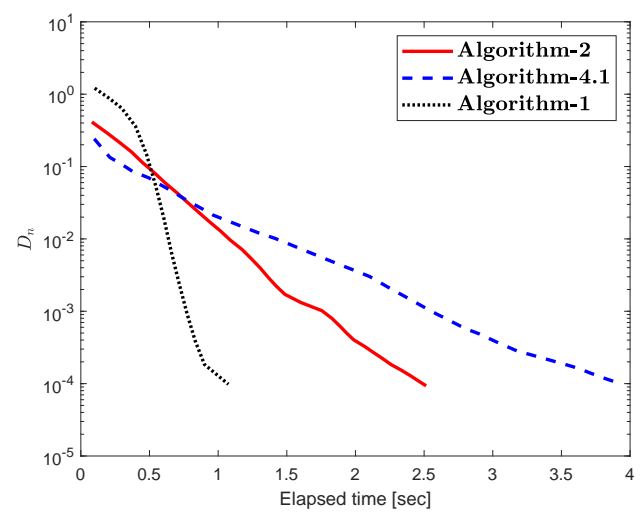

(b)

Figure 9: Numerical comparison behaviour of Algorithm 1 with Algorithm 4.1 in [22] and Algorithm 2 in [15] when $u_{0}=-2 e^{t} \cos (t)$.

Table 2: Numerical results values for Figures 6-9.

\begin{tabular}{cccc|ccc}
\hline & \multicolumn{3}{c}{ Number of iterations } & \multicolumn{3}{c}{ CPU time in seconds } \\
\hline $\mathrm{u}_{0}$ & Algorithm-2 & Algorithm-4.1 & Algorithm-1 & Algorithm-2 & Algorithm-4.1 & Algorithm-1 \\
\hline $3 \mathrm{t}^{2}$ & 28 & 36 & 19 & 1.1741502 & 2.1310576 & 1.3805529 \\
$-2 t+5 t^{2}$ & 31 & 46 & 13 & 1.689667 & 3.3207762 & 0.889326 \\
$2 e^{t} \sin (t)$ & 41 & 51 & 26 & 1.7857041 & 3.3381265 & 1.8077202 \\
$-2 e^{t} \cos (t)$ & 33 & 51 & 13 & 2.5144959 & 3.9310611 & 1.0745534 \\
\hline
\end{tabular}

\section{Conclusion}

We have designed an explicit Halpern-type extragradient method to solve pseudo-monotone equilibrium problem in a real Hilbert space, and we also confirm that the generated sequence is strongly convergent to the solution. The applications of main results are being discussed to solve particular classes of equilibrium problems. Numerical conclusions have been drawn to explain the numerical efficiency of our algorithms compared to other methods. These numerical studies have shown that viscosity effects improve the efficiency of the iterative sequence in this context.

\section{Acknowledgement}

The authors are heartily grateful to the reviewers for their valuable remarks which greatly improved the results and presentation of the paper.

This project was supported by Rajamangala University of Technology Phra Nakhon (RMUTP).

\section{References}

[1] M. Abbas, H. Iqbal, Two inertial extragradient viscosity algorithms for solving variational inequality and fixed point problems, J. Nonlinear Var. Anal., 4 (2020), 377-398. 1

[2] A. S. Antipin, Equilibrium programming: proximal methods, Comput. Math. Math. Phys., 37 (1997), 1285-1296. 1

[3] H. H. Bauschke, The approximation of fixed points of compositions of nonexpansive mappings in Hilbert space, J. Math. Anal. Appl., 202 (1996), 150-159. 1

[4] H. H. Bauschke, P. L. Combettes, Convex Analysis and Monotone Operator Theory in Hilbert Spaces, Springer, Cham, (2017). 2.2, 2.5, 5.2 
[5] M. Bianchi, S. Schaible, Generalized monotone bifunctions and equilibrium problems, J. Optim. Theory Appl., 90 (1996), 31-43. 2

[6] G. Bigi, M. Castellani, M. Pappalardo, M. Passacantando, Existence and solution methods for equilibria, European J. Oper. Res., 227 (2013), 1-11. 1

[7] E. Blum, W. Oettli, From optimization and variational inequalities to equilibrium problems, Math. Student, 63 (1994), 123-145. 1, 1, 2

[8] F. E. Browder, W. V. Petryshyn, Construction of fixed points of nonlinear mappings in Hilbert space, J. Math. Anal. Appl., 20 (1967), 197-228. 4

[9] Y. Censor, A. Gibali, S. Reich, The subgradient extragradient method for solving variational inequalities in Hilbert space, J. Optim. Theory Appl., 148 (2011), 318-335. 1

[10] Y. Censor, A. Gibali, S. Reich, Strong convergence of subgradient extragradient methods for the variational inequality problem in Hilbert space, Optim. Methods Softw., 26 (2011), 827-845. 1

[11] K. Fan, A minimax inequality and applications, Academic Press, New York, (1972). 1, 1

[12] S. D. Flåm, A. S. Antipin, Equilibrium programming using proximal-like algorithms, Math. Program., 78 (1997), $29-41$. 1

[13] F. Giannessi, A. Maugeri, P. M. Pardalos, Equilibrium problems: nonsmooth optimization and variational inequality models, Springer Science \& Business Media, New York, (2006). 1

[14] B. Halpern, Fixed points of nonexpanding maps, Bull. Amer. Math. Soc., 73 (1967), 957-961. 1, 3

[15] D. V. Hieu, Halpern subgradient extragradient method extended to equilibrium problems, Rev. R. Acad. Cienc. Exactas Fís. Nat. Ser. A Mat. RACSAM, 111 (2017), 823-840. 1, 1, 3, 5.1, 1, 2, 3, 4, 5, 5.2, 6, 7, 8, 9

[16] D. V. Hieu, Y. J. Cho, Y.-B. Xiao, Modified extragradient algorithms for solving equilibrium problems, Optimization, 67 (2018), 2003-2029. 1

[17] D. V. Hieu, Y. J. Cho, Y.-B. Xiao, P. Kumam, Modified extragradient method for pseudomonotone variational inequalities in infinite dimensional Hilbert spaces, Vietnam J. Math., 2020 (2020), 1-19.

[18] D. V. Hieu, A. Gibali, Strong convergence of inertial algorithms for solving equilibrium problems, Optim. Lett., 14 (2020), 1817-1843.

[19] D. V. Hieu, P. K. Quy, L. T. Hong, L. V. Vy, Accelerated hybrid methods for solving pseudomonotone equilibrium problems, Adv. Comput. Math., 46 (2020), 24 pages. 1

[20] D. V. Hieu, P. K. Quy, L. V. Vy, Explicit iterative algorithms for solving equilibrium problems, Calcolo, 56 (2019), 21 pages. 1

[21] D. V. Hieu, J. J. Strodiot, Strong convergence theorems for equilibrium problems and fixed point problems in Banach spaces, J. Fixed Point Theory Appl., 20 (2018), 32 pages. 1

[22] D. V. Hieu, J. J. Strodiot, L. D. Muu, Strongly convergent algorithms by using new adaptive regularization parameter for equilibrium problems, J. Comput. Appl. Math., 376 (2020), 21 pages. 5.1, 1, 2, 3, 4, 5, 5.2, 6, 7, 8, 9

[23] G. Korpelevich, The extragradient method for finding saddle points and other problems, Matecon, 12 (1976), 747-756. 1

[24] R. Kraikaew, S. Saejung, Strong convergence of the Halpern subgradient extragradient method for solving variational inequalities in Hilbert spaces, J. Optim. Theory Appl., 163 (2014), 399-412. 1

[25] S. I. Lyashko, V. V. Semenov, A new two-step proximal algorithm of solving the problem of equilibrium programming, in Optimization and Its Applications in Control and Data Sciences, Springer, Cham, 2016 (2016), 315-325. 1

[26] P.-E. Maingé, Strong convergence of projected subgradient methods for nonsmooth and nonstrictly convex minimization, Set-Valued Anal., 16 (2008), 899-912. 1, 2.4

[27] G. Mastroeni, On auxiliary principle for equilibrium problems, in Nonconvex Optimization and Its Applications, Kluwer Acad. Publ., Norwell, MA, 2003 (2003), 289-298. 2

[28] L. D. Muu, W. Oettli, Convergence of an adaptive penalty scheme for finding constrained equilibria, Nonlinear Anal., 18 (1992), 1159-1166. 1

[29] S. Regmi, I. K. Argyros, S. Georg, Direct comparison between two third convergence order schemes for solving equations, Symmetry, 12 (2020), 10 pages. 1

[30] H. U. Rehman, P. Kumam, A. B. Abubakar, Y. J. Cho, The extragradient algorithm with inertial effects extended to equilibrium problems, Comput. Appl. Math., 39 (2020), 26 pages. 1

[31] H. U. Rehman, P. Kumam, I. K. Argyros, N. A. Alreshidi, W. Kumam, W. Jirakitpuwapat, A self-adaptive extragradient methods for a family of pseudomonotone equilibrium programming with application in different classes of variational inequality problems, Symmetry, 12 (2020), 27 pages.

[32] H. U. Rehman, P. Kumam, I. K. Argyros, W. Deebani, W. Kumam, Inertial extra-gradient method for solving a family of strongly pseudomonotone equilibrium problems in real Hilbert spaces with application in variational inequality problem, Symmetry, 12 (2020), 24 pages.

[33] H. U. Rehman, P. Kumam, I. K. Argyros, M. Shutaywi, Z. Shah, Optimization based methods for solving the equilibrium problems with applications in variational inequality problems and solution of nash equilibrium models, Mathematics, 8 (2020), 28 pages.

[34] H. U. Rehman, P. Kumam, Y. J. Cho, Y. I. Suleiman, W. Kumam, Modified Popov's explicit iterative algorithms for solving pseudomonotone equilibrium problems, Optim. Methods Softw., 36 (2021), 82-113.

[35] H. U. Rehman, P. Kumam, Y. J. Cho, P. Yordsorn, Weak convergence of explicit extragradient algorithms for solving equilibirum problems, J. Inequal. Appl., 2019 (2019), 1-25. 
[36] H. U. Rehman, P. Kumam, W. Kumam, M. Shutaywi, W. Jirakitpuwapat, The inertial sub-gradient extra-gradient method for a class of pseudo-monotone equilibrium problems, Symmetry, 12 (2020), 1-25. 1

[37] R. T. Rockafellar, Convex analysis, Princeton University Press, Princeton, (1970). 2.1

[38] G. Stampacchia, Formes bilinéaires coercitives sur les ensembles convexes, C. R. Acad. Sci. Paris, 258 (1964), $4413-4416$. 4

[39] D. Q. Tran, M. L. Dung, V. H. Nguyen, Extragradient algorithms extended to equilibrium problems, Optimization, 57 (2008), 749-776. 1, 3, 3, 5.1

[40] L. Wang, L. Yu, T. Li, Parallel extragradient algorithms for a family of pseudomonotone equilibrium problems and fixed point problems of nonself-nonexpansive mappings in Hilbert space, J. Nonlinear Funct. Anal., 2020 (2020), 1-16. 1

[41] H.-K. Xu, Another control condition in an iterative method for nonexpansive mappings, Bull. Austral. Math. Soc., 65 (2002), 109-113. 2.3 